\title{
Dimensional regulation of cell-cycle events in Escherichia coli during steady-state growth
}

\author{
N. B. Grover ${ }^{1}$ and C. L. Woldringh ${ }^{2}$ \\ Author for correspondence: N. B. Grover. Tel: +972 2675 8360. Fax: +972 26436890. \\ e-mail: norman@md.huji.ac.il
}

\footnotetext{
1 Hubert H. Humphrey Center for Experimental Medicine and Cancer Research, Hebrew University, Faculty of Medicine, PO Box 12272 . Jerusalem 91120, Israel

2 Section of Molecular Cytology, Institute for Molecular Cell Biology, University of Amsterdam, BioCentrum, Kruislaan 316, 1098 SM Amsterdam, The Netherlands
}

\begin{abstract}
Two opposing models have been put forward in the literature to describe the changes in the shape of individual Escherichia coli cells in steady-state growth that take place during the cell cycle: the Length model, which maintains that the regulating dimension is cell length, and the Volume model, which asserts it to be cell volume. In addition, the former model envisages cell diameter as decreasing with length up to constriction whereas the latter sees it as being constrained by the rigid cell wall. These two models differ in the correlations they predict between the various cellular dimensions (diameter, length, volume) not only across the entire population of bacteria but also, and especially, within subpopulations that define specific cell-cycle events (division, for example, or onset of constriction); the coefficients of variation at these specific events are also expected to be very different. Observations from cells prepared for electron microscopy (air-dried) and for phase-contrast microscopy (hydrated) appeared qualitatively largely in accordance with the predictions of the Length model. To obtain a more quantitative comparison, simulations were carried out of populations defined by each of the models; again, the results favoured the Length model. Finally, in age-selected cells using membrane elution, the diameter-length and diameter-volume correlations were in complete agreement with the Length model, as were the coefficients of variation. It is concluded that, at least with respect to cell-cycle events such as onset of constriction and cell division, length rather than volume is the controlling dimension.
\end{abstract}

Keywords: cell shape during growth cycle, correlation between cell dimensions, diameter changes with cell age, length versus volume regulation, models of individual cell growth

\section{INTRODUCTION}

The bacterium Escherichia coli has often been used to study cell shape, both during the cell cycle and under different growth conditions, probably because of its relatively well-defined geometry. Thirty years ago several mathematical relationships were put forward to describe the observed changes in cell size (Donachie \& Begg, 1970; Helmstetter \& Cooper, 1968; Pritchard et al., 1969), and these were followed by various empirical expressions to account for specific changes in particular dimensions (Donachie et al., 1976; Rosenberger et al., 1978; Woldringh et al., 1990; Zaritsky et al., 1982) based on the notion that cell length (or surface area) is extended at a discrete number of growth zones that double concomitant with a well-defined event in the cell cycle. But no real biological basis was provided for the parameters used, and the interpretation of dimensional modification in terms of a mechanism for shape determination has remained elusive.

In the early work on bacterial shape and form, cell diameter was at the limit of optical resolution and so measurements concentrated on length. Cell length was seen to double during the cell cycle, whilst the diameter appeared to remain constant (Schaechter et al., 1958, 1962). Later, electron-microscopic studies confirmed the essential findings and reported only small, apparently random fluctuations in diameter (Harvey \& Marr, 1966). Trueba \& Woldringh (1980) observed that the average diameter of slow-growing cells decreased gradually as a function of cell length prior to constriction, rising again during the constriction process; this was later corroborated by Vardi \& Grover (1992) in an 


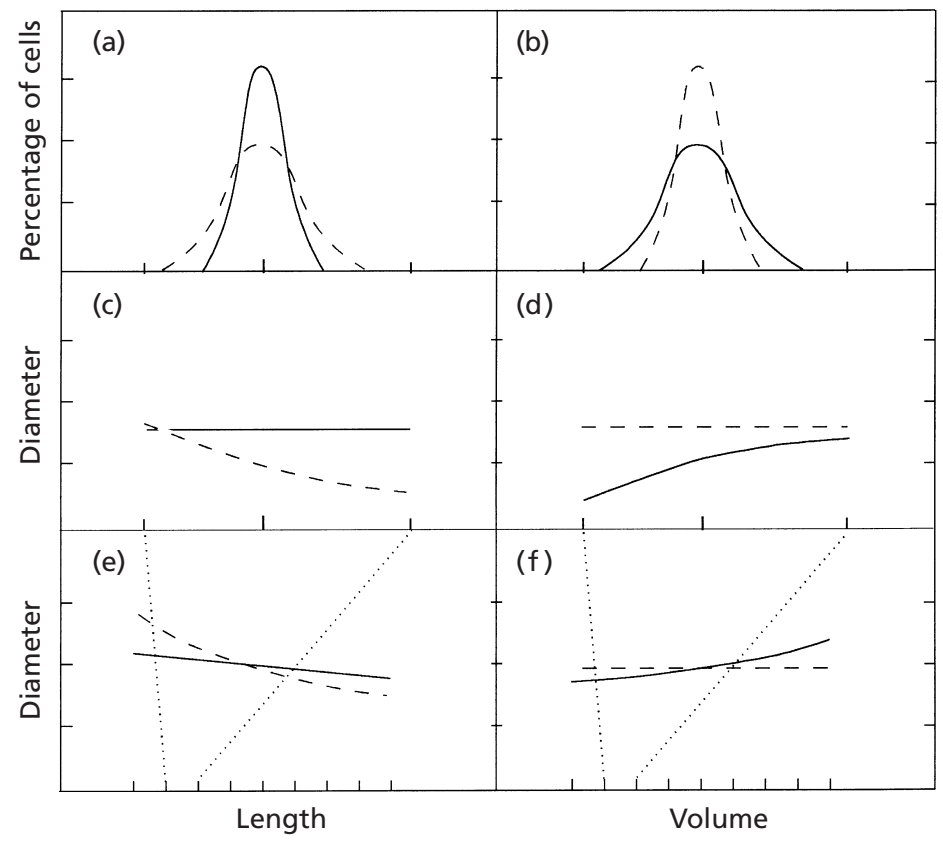

Fig. 1. Schematic drawing of size distributions (a, b) and functional dependence of cell diameter on cell length (c, e) and volume $(d, f)$ at a particular cellcycle event such as birth (a-d) and for the entire nonconstricting population $(e, f)$. The curvature of each non-linear regression line is displayed with its correct sign, but magnitudes have been exaggerated for purposes of illustration; slopes are not to scale. Solid lines, Length model; dashed lines, Volume model. Note that the lines in the lower panels do not necessarily imply that the diameters of individual cells change systematically during their cell cycle (see text). A three-dimensional rendition of this figure in colour can be viewed at http://mic. sgmjournals.org/cgi/data/147/1/171/DC1/1.

elaborate analysis of more than 55000 cells. Trueba \& Woldringh (1980) inferred from their findings that the diameter of individual cells decreases systematically during their elongation, possibly because of the maintenance of a constant surface-to-volume ratio during the cell cycle.

This interpretation of the observed negative correlation between cell diameter and cell length was challenged by Cooper (1989), who proposed instead that elongating $E$. coli bacteria maintain a constant diameter throughout the cell cycle: because the cell wall serves as a constraining hoop, the cells can only change their diameter slowly, over the course of several generations. In this view, individual cells are assumed to initiate the various cell-cycle events at essentially the same volume and to accommodate any variation in width by a compensating change in length. Thus cells that happen to be wider than average will initiate DNA replication or cell division when they are relatively shorter, whereas narrower cells will do so when they are longer. It is this reciprocal relationship at fixed cell volumes that produces the overall negative correlation between cell diameter and cell length even though actual cell diameter at the level of the individual bacterium does not change during the cell cycle (Cooper, 1991).

These two approaches give rise to two opposing models of shape regulation that make very different predictions about the variations in cell dimensions at a regulated cell-cycle event and about correlations between the various dimensions. The models can be described as (i) a Length model (Fig. 1, solid lines), in which cell length is the regulating dimension and so displays only minimal variation at any particular cell-cycle event (cell birth, for example: narrow distribution in Fig. 1a but broad distribution in Fig. 1b), and (ii) a Volume model (Fig. 1, dashed lines), in which volume is the regulating di- mension and so displays minimal variation at cell-cycle events (narrow distribution in Fig. 1b but broad distribution in Fig. 1a).

In the Length model, which could be termed a 'variable diameter' model, it is assumed that during growth the individual cells change in diameter systematically (solid line in Fig. 1e) but show no correlation between diameter and length at any particular cell-cycle event (solid line in Fig. 1c). Fluctuations in cell diameter are therefore accompanied by corresponding changes in volume, causing a strong positive correlation between the two (solid line in Fig. 1d).

In the Volume model, which could be termed a 'constrained hoop' model, it is assumed that during growth the individual cells maintain a constant diameter imposed by a rigid cell wall (dashed line in Fig. 1f) and show no correlation between diameter and volume at any particular cell-cycle event (dashed line in Fig. 1d). Fluctuations in cell diameter are therefore accompanied by compensatory changes in length, causing a strong negative correlation between the two (dashed line in Fig. 1c).

Clearly, the Length model and the Volume model make very different predictions about the correlations between the various dimensions of the cell and about the variations in these dimensions at all regulated cell-cycle events. We have therefore examined both previously published (Vardi \& Grover, 1992) and newly acquired data in the light of these two models and carried out extensive simulation studies in an attempt to distinguish between them.

\section{METHODS}

Organism. E. coli B/r A cells (ATCC 12407) were used in all experiments. 


\section{Table 1. List of symbols}

Symbols that are introduced solely to simplify the layout of an expression and are not used elsewhere, do not appear in this list.

\begin{tabular}{|c|c|}
\hline Symbol & Definition \\
\hline$a$ & Cell age (in units of $\bar{\tau}$ ) \\
\hline$a_{1}$ & Age of youngest cell in subpopulation \\
\hline$a_{2}$ & Age of oldest cell in subpopulation \\
\hline $\mathrm{CV}$ & Coefficient of variation (standard deviation $\div$ mean) \\
\hline $\mathrm{CV}_{\mathrm{D}}$ & CV of cell diameter $D$ \\
\hline $\mathrm{CV}_{\mathrm{Da}}$ & CV of cell diameter $D$ at age $a$ \\
\hline $\mathrm{CV}_{\mathrm{L}}$ & CV of cell length $L$ \\
\hline $\mathrm{CV}_{\mathrm{La}}$ & $\mathrm{CV}$ of cell length $L$ at age $a$ \\
\hline $\mathrm{CV}_{\mathrm{V}}$ & CV of cell volume $V$ \\
\hline $\mathrm{CV}_{\mathrm{Va}}$ & $\mathrm{CV}$ of cell volume $V$ at age $a$ \\
\hline $\mathrm{CV}_{\tau}$ & $\mathrm{CV}$ of interdivision time $\tau$ \\
\hline$D$ & Cell diameter \\
\hline$D^{\prime}$ & Intermediate variable defined as $z_{1} \rho^{\prime}+z_{2}\left(1-\rho^{\prime 2}\right)^{1 / 2}$ \\
\hline $\bar{D}$ & Mean cell diameter \\
\hline$D(a)$ & Cell diameter $D$ at age $a$ \\
\hline$f(\tau)$ & Distribution of interdivision times $\tau$ \\
\hline$i$ & Running cell count during simulation \\
\hline$L$ & Cell length \\
\hline $\bar{L}$ & Mean cell length \\
\hline$L(a)$ & Cell length $L$ at age $a$ \\
\hline $\bar{L}(a)$ & Mean cell length at age $a$ \\
\hline$n$ & Sample size \\
\hline$P$ & Probability that null hypothesis is true \\
\hline$r$ & Correlation coefficient \\
\hline$r_{\mathrm{DL}}$ & Correlation between diameter $D$ and length $L$ \\
\hline$r_{\mathrm{DV}}$ & Correlation between diameter $D$ and volume $V$ \\
\hline$r_{\mathrm{LV}}$ & Correlation between length $L$ and volume $V$ \\
\hline SD & Standard deviation \\
\hline$u$ & $\begin{array}{l}\text { Random number from a standard uniform } \\
\text { distribution }\end{array}$ \\
\hline V & Cell volume \\
\hline $\bar{V}$ & Mean cell volume \\
\hline$V(a)$ & Cell volume $V$ at age $a$ \\
\hline $\bar{V}(a)$ & Mean cell volume at age $a$ \\
\hline$z_{1}, z_{2}, z_{3}$ & $\begin{array}{l}\text { Random numbers from a standard normal } \\
\text { distribution }\end{array}$ \\
\hline$v(a)$ & Steady-state age distribution \\
\hline$\xi$ & Width of constriction region \\
\hline$\rho$ & $\begin{array}{l}\text { Diameter-length correlation coefficient in } \\
\text { simulated population }\end{array}$ \\
\hline$\rho^{\prime}$ & Intermediate variable defined as $\rho \mathrm{CV}_{\mathrm{L}} / \mathrm{CV}_{\mathrm{La}}$ \\
\hline$\tau$ & Cell interdivision time \\
\hline $\bar{\tau}$ & Mean cell interdivision time \\
\hline
\end{tabular}

Growth conditions. Cells were grown in batch cultures using either MOPS medium (MOPS supplemented with $0 \cdot 1 \%$ Lalanine; Sigma) to obtain a doubling time of $113 \mathrm{~min}$, or in glucose minimal medium (Helmstetter \& Cooper, 1968) to obtain a doubling time of $42 \mathrm{~min}$. Steady-state growth was reached after culturing the cells for at least 10 generations at the same growth rate whilst maintaining cell concentration below $10^{7}$ cells $\mathrm{ml}^{-1}$ by periodic dilutions. Cells were deemed to have attained steady state when either average cell size, as measured by an electronic particle counter, or their length distributions, as measured by image cytometry, no longer changed with time.

For age selection, cells in steady-state growth (doubling time $42 \mathrm{~min}$ ) were poured over a nitrocellulose filter for the elution of baby cells according to the membrane elution technique (Helmstetter \& Cooper, 1968).

Sample preparation. For electron microscopy, cells were fixed by adding $\mathrm{OsO}_{4}$ to the growth medium at a final concentration of $0.1 \%$, and prepared using the agar filtration method (Woldringh et al., 1977). For phase-contrast microscopy, samples from the eluted suspension of baby cells and from the original, steady-state culture were fixed by adding formaldehyde to the growth medium at a final concentration of $0 \cdot 25 \%$. The cells were then sent from Florida to Amsterdam where they were postfixed by adding $\mathrm{OsO}_{4}$ at a final concentration of $0 \cdot 1 \%$; the time between fixations was $6 \mathrm{~d}$. Finally, the cells were prepared in their hydrated state on a thin slab of $1.5 \%$ agarose as described previously (Van Helvoort et al., 1998). Sample size for phase-contrast microscopy was 488 for membrane-eluted cells and 1141 for steady-state cells.

Microscopy and data acquisition. The agar filters were photographed in focus on $70 \mathrm{~mm}$ film (SD-281; Kodak) using a transmission electron microscope (EM300; Philips) at $60 \mathrm{kV}$ with a final magnification of $\times 3600$; exposure time was $1.5 \mathrm{~s}$. The electron micrographs were input to an image processing system (System 100; Liacom) controlled by a computer (PDP$11 / 23$; Digital Equipment), with a spatial resolution of $0.004 \mu \mathrm{m} \mathrm{pixel}^{-1}$ and 256 grey levels. The system (Vardi, 1990) recognizes the cells on the micrographs and detects their boundaries automatically by means of a multi-thresholding method that locates edges along the maximum optical density gradient. The cells are characterized using a syntactical approach and their morphological properties identified, on the basis of which the various cell dimensions are then calculated. These calculations require no geometrical assumptions other than that the cell be locally radially symmetrical along its midline; thus, the dimensions of a cell reflect its actual shape rather than the usual idealizations.

A total of 55269 cells were recorded in this way, with a mean diameter \pm SD of $0.447 \pm 0.034 \mu \mathrm{m}$, a mean length of $1.36 \pm 0.29 \mu \mathrm{m}$ and a mean volume of $0.186 \pm 0.051 \mu \mathrm{m}^{3}$. (SD refers to the standard deviation; a complete list of symbols used appears in Table 1). Dividing and newborn cells were obtained from the deeply constricted cells and from their prospective daughters, respectively, chosen so that the width of the constricted region $\xi$ did not exceed $50 \%$ of the mean width of the prospective daughter cells. (This threshold, $0 \cdot 50$, was based on the smallest value that still gave a reasonable sample size, 637 constricting cells or 1274 prospective daughters; below that level, the number of constricted cells falls off rapidly.) Constriction was taken to begin at a relative $\xi$ of 0.79 (chosen for the abrupt increase in cell length beyond that point), resulting in 50796 nonconstricting cells (92\%); all cells with relative $\xi$ in the inclusive range $0.65-0.78$ were considered to be in constriction onset. (This interval was selected so as to be as narrow as possible consistent with providing an adequate number of cells, 688.)

Digital images from the hydrated cells of either the glucose or the alanine cultures were acquired with a cooled chargecoupled device (CCD) camera at a resolution of $1317 \times 1035$ pixels (RTE-1317-k-1; Princeton Instruments) mounted on a 
conventional fluorescence microscope (BH-2; Olympus) equipped with a $\times 100$ SPlan PL phase-contrast objective (n.a. 1.25). To determine cell dimensions, the cell contour was first demarcated by creating a maximum gradient image (using noise reduction, magnification and Sobel filtering) and then thresholding and skeletonizing. The major axis of the bestfitting ellipse of each cell was chosen to sample the length within the contour; the diameter was sampled along the three lines perpendicular to the axis at 25,50 and $75 \%$, and the largest one selected. Here, cell volume was computed in the usual manner: by assuming an idealized geometry of a right circular cylinder with hemispherical polar caps.

\section{RESULTS}

\section{Variation at specific events}

In the Volume model, cell volume is the regulating dimension and any variation in diameter is compensated for by a corresponding change in length. As a result, the coefficients of variation (CV) of length (dashed distribution in Fig. 1a) and of diameter are expected to be larger than that of volume (dashed distribution in Fig. 1b). The reverse holds true for the Length model (solid distributions). In Table 2, the differences of the predicted CVs are compared with the experimental observations: in all cases, the coefficients of variation of cell volume $\left(\mathrm{CV}_{\mathrm{V}}\right)$ significantly exceed those of length $\left(\mathrm{CV}_{\mathrm{L}}\right)$ and diameter $\left(\mathrm{Cv}_{\mathrm{D}}\right)$. Clearly, the Length model is favoured, and quite strongly.

\section{Correlations between cell dimensions}

In addition to different cvs at specific cell-cycle events, the two models also predict different correlations between the various dimensions. Schematic representations of the diameter-length correlations $r_{\mathrm{DL}}$ and the diameter-volume correlations $r_{\mathrm{DV}}$ as predicted by each of the models are indicated in Fig. 1. Subpopulations of cells selected at particular cell-cycle events provide a sensitive means of discriminating between the models. In addition to selecting by microscopic measurement, therefore, we have included a sample of membraneeluted baby cells (kindly provided by C. E. Helmstetter, Florida Institute of Technology, Melbourne, FL, USA). Scatter plots of diameter versus length and diameter versus volume are shown in Fig. 2 together with the least-squares regression lines. It is clear that the babycell data support the Length model, in most cases even more strongly than do the microscopically selected cells (see below).

In Table 3, the theoretical predictions are compared with experimental values obtained from the electronmicroscopic measurements of Vardi \& Grover (1992). To make sure that the shape changes introduced by the dehydration of cells during agar filtration (Vardi \& Grover, 1993) are not relevant in the present context, we also looked at a sample of newborn and steady-state hydrated cells using phase-contrast microscopy. The results (Tables 2 and 3) are in good agreement with those from the dehydrated sample and suggest that whatever inferences we draw from the latter will reflect actual cell properties rather than preparation artifacts.

The experimental observations are clearly in accordance with the predictions of the Length model as regards both particular cell-cycle events (birth, onset of constriction, division in Table 3; birth in Fig. 2) and the entire population of nonconstricting cells (Table 3, last two lines). A weak negative correlation between diameter and length in nonconstricting cells has also been reported by Trueba \& Woldringh (1980) for two E. coli

Table 2. Coefficients of variation (CV) predicted by the Length ( $L$ ) and Volume (V) models, and observed experimentally

\begin{tabular}{|c|c|c|c|c|c|c|c|c|}
\hline \multirow[t]{2}{*}{ Subpopulation } & \multirow{2}{*}{$\begin{array}{r}\text { Size (no. } \\
\text { of cells) }\end{array}$} & \multicolumn{2}{|c|}{ Predicted $\mathrm{CV}_{\mathrm{L}}-\mathrm{CV}_{\mathrm{V}}$} & \multicolumn{2}{|c|}{ Predicted $\mathrm{CV}_{\mathrm{D}}-\mathrm{CV}_{\mathrm{V}}$} & \multicolumn{3}{|c|}{ Experimental $\mathrm{CV}^{*}$} \\
\hline & & $L$ model & $V$ model & $L$ model & $V$ model & $L$ & $D$ & $V$ \\
\hline Newborn $†$ & 1274 & $<0$ & $>0$ & $<0$ & $>0$ & $0 \cdot 09$ & $0 \cdot 08$ & $0 \cdot 17$ \\
\hline Newborn $\neq$ & 488 & $<0$ & $>0$ & $<0$ & $>0$ & $0 \cdot 12$ & $0 \cdot 06$ & $0 \cdot 18$ \\
\hline Onset of constriction $†$ & 688 & $<0$ & $>0$ & $<0$ & $>0$ & $0 \cdot 08$ & $0 \cdot 06$ & $0 \cdot 15$ \\
\hline Dividing $†$ & 637 & $<0$ & $>0$ & $<0$ & $>0$ & $0 \cdot 09$ & $0 \cdot 07$ & $0 \cdot 16$ \\
\hline All nonconstricting $\dagger$ & 50796 & $<0$ & $>0$ & $<0$ & $<0$ & $0 \cdot 19$ & $0 \cdot 08$ & $0 \cdot 27$ \\
\hline All nonconstricting $\mathbb{S}$ & 1141 & $<0$ & $>0$ & $<0$ & $<0$ & $0 \cdot 15$ & $0 \cdot 05$ & $0 \cdot 20$ \\
\hline
\end{tabular}

*Differences between $\mathrm{CV}_{\mathrm{V}}$ and corresponding $\mathrm{CV}_{\mathrm{L}}$ and $\mathrm{CV}_{\mathrm{D}}$ are very highly significant $(P<0 \cdot 001)$ in all cases, as determined by the test proposed by Miller (Zar, 1999).

† Microscopically selected cells: data obtained by Vardi \& Grover (1992) from electron-microscopic measurements of cells growing in steady state (doubling time $113 \mathrm{~min}$ ).

¥ Selected by membrane elution: data obtained from phase-contrast microscopic measurements; scatter plot presented in Fig. 2.

$\mathbb{S}$ Obtained from phase-contrast microscopic measurements of cells growing in steady state (doubling time $42 \mathrm{~min}$ ). 


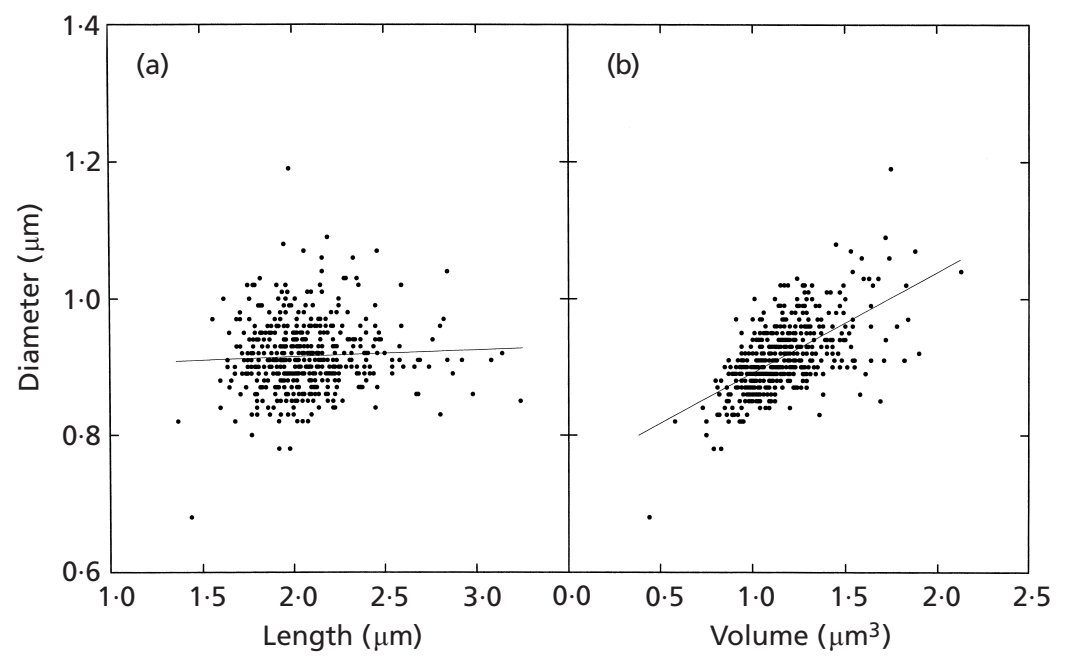

Fig. 2. Scatter plot of diameter versus length (a) and diameter versus volume (b) of a sample of $488 \mathrm{E}$. coli $\mathrm{B} / \mathrm{r} A$ cells selected by membrane elution (Helmstetter \& Cooper, 1968) and prepared for phase-contrast microscopy as described in Methods, together with the corresponding leastsquares regression lines. The slope of the diameter-length line is not significantly different from zero $(P>0.25)$; the slope of the diameter-volume line is, very much so $(P<0.001)$.

Table 3. Correlation coefficients predicted by the Length $(L)$ and Volume $(V)$ models, and observed experimentally

\begin{tabular}{|c|c|c|c|c|c|c|c|c|c|}
\hline \multirow[t]{2}{*}{ Subpopulation } & \multirow{2}{*}{$\begin{array}{l}\text { Size (no. } \\
\text { of cells) }\end{array}$} & \multicolumn{4}{|c|}{ Diameter-length correlation $\dagger$} & \multicolumn{4}{|c|}{ Diameter-volume correlation $\dagger$} \\
\hline & & $L$ model & $V$ model & Experimental & $P \neq$ & $L$ model & $V$ model & Experimental & $P \neq$ \\
\hline Newborn $\$$ & 1274 & 0 & $<0$ & $-0 \cdot 117$ & $* * *$ & $>0$ & 0 & $+0 \cdot 410$ & $* * *$ \\
\hline Newborn $\|$ & 488 & 0 & $<0$ & $+0 \cdot 050$ & NS & $>0$ & 0 & $+0 \cdot 602$ & $* * *$ \\
\hline Onset of constriction $\mathbb{S}$ & 688 & 0 & $<0$ & $+0 \cdot 048$ & NS & $>0$ & 0 & $+0 \cdot 805$ & $* * *$ \\
\hline Dividing $\mathbb{S}$ & 637 & 0 & $<0$ & $+0 \cdot 007$ & NS & $>0$ & 0 & +0.787 & $* * *$ \\
\hline All nonconstricting $\mathbb{S}$ & 50796 & $<0$ & $<0$ & $-0 \cdot 120$ & $\because * *$ & $>0$ & 0 & +0.336 & $* * *$ \\
\hline All nonconstricting & 1141 & $<0$ & $<0$ & -0.076 & $* *$ & $>0$ & 0 & $+0 \cdot 397$ & $\because * *$ \\
\hline
\end{tabular}

† See schematic regression lines in Fig. 1.

$\ddagger$ Probability that population correlation coefficient is zero (alternative hypothesis negative for diameter-length correlations, positive for diameter-volume correlations): Ns, $P>0.05 ; *, 0.05>P>0.01 ; * *, 0.01>P>0.001 ; * * *, P<0.001$.

$\$$ Microscopically selected cells: data obtained by Vardi \& Grover (1992) from electron-microscopic measurements of cells growing in steady state (doubling time $113 \mathrm{~min}$ ).

|| Selected by membrane elution: data obtained from phase-contrast microscopic measurements; scatter plot presented in Fig. 2.

g Obtained from phase-contrast microscopic measurements of cells growing in steady state (doubling time $42 \mathrm{~min}$ ).

$\mathrm{B} / \mathrm{r}$ strains at slow growth rates, in agreement with both models.

\section{Computation by simulation}

To obtain a more quantitative comparison between the two models, simulations on populations of $10^{6}$ cells were carried out as described in the Appendix, with parameter values based on actual data (Vardi \& Grover, 1992) wherever possible; the results are summarized in Table 4. The choices for $\mathrm{cv}_{\mathrm{D}}$ were derived from the experimental observations: Vardi \& Grover (1992) found $7.6 \%$ and Trueba \& Woldringh (1980) reported $10 \cdot 7 \%$.

The Volume model requires that $r_{\mathrm{DV}}$ be zero; based on that, the simulated value for $r_{\mathrm{DL}}(-0.396)$ is very much higher (in absolute value) than that observed experimentally $(-0 \cdot 120)$ even with $\mathrm{CV}_{\mathrm{D}}$ as low as $5 \%$. The
Length model takes as input the observed value for $r_{\mathrm{DL}}$; the predicted $r_{\mathrm{DV}} \mathrm{s}(+0.277$ and +0.556$)$ bracket the observed value $(+0 \cdot 336)$. The length-volume correlations predicted by the two models are very similar and cannot be used to distinguish between them, because most of the variance in $L$ and $V$ is common to them both owing to their concomitant increase as the cell grows, regardless of the specifics of any particular model.

The change in $r_{\mathrm{DL}}$ with $\mathrm{CV}_{\mathrm{D}}$ in the Volume model is not unexpected. As the coefficient of variation of cell diameter $\mathrm{CV}_{\mathrm{D}}$ decreases, the length-volume correlation increases until, for constant diameter $D$, it approaches unity. This follows directly from the assumed relationship between length and volume: $V(a)=$ $\frac{1}{4} \pi D^{2}\left[\bar{L}(a)-\frac{1}{3} D\right]$. (It will always be somewhat less than 1 , however, because $r_{\mathrm{LV}}$ is actually the correlation between the volume of a cell $V(a)$ and its length $L(a)$, not the mean length at that age $\bar{L}(a)$, and $L(a)$ is taken to be 
Table 4. Correlation coefficients for entire population of nonconstricting cells produced by computer simulations of the Length $(L)$ and the Volume $(V)$ models for two values of the coefficient of variation of cell diameter $\left(\mathrm{Cv}_{\mathrm{D}}\right)$

\begin{tabular}{|c|c|c|c|c|c|}
\hline \multirow[t]{2}{*}{ Correlation } & \multicolumn{2}{|c|}{$\mathrm{CV}_{\mathrm{D}} \mathbf{0 \cdot 0 5}$} & \multicolumn{3}{|c|}{$\mathrm{CV}_{\mathrm{D}} \mathbf{0} \cdot 10$} \\
\hline & $L$ model $*$ & $V$ model $\dagger$ & $L$ model* & $V$ model $\uparrow$ & Experimental \\
\hline Diameter-length & $-0 \cdot 12 \mathbb{S}$ & -0.396 & $-0 \cdot 12 \mathbb{S}$ & -0.649 & $-0 \cdot 120$ \\
\hline Diameter-volume & $+0 \cdot 277$ & $0 \mathbb{S}$ & +0.556 & $0 \mathbb{S}$ & $+0 \cdot 336$ \\
\hline Length-volume & +0.822 & $+0 \cdot 840$ & +0.663 & +0.671 & +0.838 \\
\hline
\end{tabular}

${ }^{*} \mathrm{CV}_{\mathrm{L}}$ at controlled cell-cycle event $=7 \cdot 5 \%, \mathrm{CV}_{\mathrm{V}}$ due to experimental error $=10 \%$; see Appendix for details.

$+\mathrm{CV}_{\mathrm{L}}$ due to experimental error $=7 \cdot 5 \%, \mathrm{CV}_{\mathrm{V}}$ at controlled cell-cycle event $=10 \%$; see Appendix for details.

‡ Microscopically selected cells: data obtained by Vardi \& Grover (1992) from electron-microscopic measurements of cells growing in steady state (doubling time $113 \mathrm{~min}$ ).

\Component of respective model.

randomly distributed about $\bar{L}(a)$ to allow for experimental error, as described in the Appendix.) Since $r_{\mathrm{LV}}^{2}$ is the proportion of variance in $L$ accounted for by $V$, as $\mathrm{CV}_{\mathrm{D}}$ decreases there is less and less unique variance left to be attributed to the diameter and eventually, with $D$ constant and $r_{\mathrm{LV}}$ at its maximum (and $r_{\mathrm{DV}}$ set at 0 , as required by the Volume model), $r_{\mathrm{DL}}$ approaches zero. Similar arguments apply to $r_{\mathrm{DV}}$ in the Length model.

\section{DISCUSSION}

The present analysis of the dimensions of E. coli cells in steady-state growth prepared either in the dried state for electron microscopy or in the hydrated state for light microscopy, indicates that the dimensional changes taking place during the cell cycle are better described by the Length model than by the Volume model, and that the dimension regulating cell-cycle events is length rather than volume.

A regulating dimension is defined here as one that presents a structural or biochemical constraint to an underlying control mechanism performing an essential step in the cell cycle. Stating that a particular dimension (length or volume, depending on the model) regulates cell-cycle events in steady-state growth is insufficient by itself to allow quantitative predictions of cell shape. More information is required.

Cooper (1989) augmented his original specification of control by cell volume, inspired by Koch \& Schaechter (1962), with that of a constant diameter imposed on a growing cell by a rigid wall, the constraining hoops of the peptidoglycan chains. Trueba \& Woldringh (1980) added to control by cell length their empirical observation that diameter decreases with cell length up to constriction; instead of restraining peptidoglycan molecules, they suggested that shape was perhaps determined by a constant surface-to-volume ratio. Such a constraint will of course provide the requisite negative correlation between diameter and length-unfortunately, it also predicts a strong negative diametervolume correlation, just the opposite of what is found experimentally (Table 4). In the present Length model, therefore, we just assume a weak negative linear diameter-length correlation without specifying its origin. The implication of this is that here, in sharp contrast to the assumption in the Volume model, an individual cell can and does change its diameter during the cell cycle.

To represent the entire population of nonconstricting cells, the contributions from all the subpopulations have to be summed. For the Length model, this leads to a positive correlation between diameter and volume (solid line in Fig. 1f) because each individual component is positive (solid line in Fig. 1d); the overall diameterlength correlation is, of course, negative as specified (solid line in Fig. 1e). The Volume model predicts a negative correlation between diameter and length (dashed line in Fig. 1e) due to the summing of the individual contributions, which are all negative (dashed line in Fig. 1c); as expected, there is no correlation between diameter and volume (dashed lines in Fig. 1d and $\mathrm{f}$ ).

Selection by dimension, any dimension, is not informative. In cells with the same length, the diameter-volume correlation will always be large and positive whereas in cells with the same volume, the diameter-length correlation will always be large and negative. This is a geometrical imperative, quite independent of any model or growth law, and applies to all shapes in which diameter and length are both necessary and sufficient to determine volume.

A curious point should be noted here. The Volume model states that the diameter of an individual cell remains constant during its cell cycle in steady-state growth. Nonetheless, the strong negative correlation at any particular event in the cycle between diameter and 
length that is inherent in this model gives rise to an apparent decrease in diameter with cell age when expressed in terms of length. Looked at differently, a negative $r_{\mathrm{DL}}$ does not necessarily imply that cell diameter decreases during the cell cycle. This effect of combining the contributions of separate subpopulations was first pointed out by Cooper (1989) and termed 'correlated variables'. The same holds true in the Length model: the positive correlation at particular cell-cycle events between diameter and volume gives rise to an apparent increase in diameter with cell age when expressed in terms of volume.

The superior fit of the Length model as regards both variation (Table 2) and correlation (Table 3), indicates that cells at particular events during their cycle exhibit constant length rather than constant volume. Why length? We have suggested previously (Woldringh et al., 1990) that constant length could reflect the amount of DNA in a non-replicating chromosome that selforganizes into a nucleoid of fixed linear dimension. During DNA replication and cell elongation, the daughter chromosomes are segregated and eventually organized into two daughter nucleoids in a cell twice the original length.

Regardless of the actual mechanism, however, what we have shown here is that cell diameter is more likely to vary systematically with cell age than to conform to the constraints of a rigid hoop. Such behaviour would be consistent with the observed ability of cell diameter to change after a nutritional shift-up (Woldringh et al., 1980) or shift-down (Zaritsky et al., 1993).

Although it seems highly unlikely that any major cellcycle event would be regulated directly by a single dimensional parameter, the magnitude of the variations in cell volume or in cell length observed at such events could shed light on the underlying biological control system. Thus, a particularly small variation in volume at initiation of DNA replication, Boye et al. (1996) report less than $9 \%$, might indicate a biochemical sensing mechanism for the concentration of an initiator protein (Hansen et al., 1991), whereas a small variation in length at a particular event, onset of constriction for example, could reflect a constraint on the displacement of the nucleoid along the cell axis (Woldringh et al., 1994).

Looked at in that way, it is not unreasonable to consider a composite model: volume regulation at initiation of chromosome replication, say, and length regulation at initiation of cell constriction. We have not been able to test this experimentally because our cells do not contain external physical markers for initiation of chromosome replication, as they do for constriction and division, and so we cannot rule it out at this stage.

\section{APPENDIX}

This Appendix describes the simulation process in detail. We begin by stating the assumptions common to the two models, then present those specific to each. These are accompanied by the various parameter values used, which are based on actual data (Vardi \& Grover, 1992) wherever possible. To test the robustness of our conclusions, we repeated the simulation using different assumptions for cell growth, cell shape and the interdivision time distribution, over a wide range of parameter values, as listed under the subheading Robustness towards the end of this Appendix. The actual numerical output of the simulations differed somewhat with each combination, usually by less than $1 \%$; in no case were the relevant implications or inferences affected.

A step-by-step description of the execution of the Length model, the more complicated of the two, is given, accompanied by an annotated flow chart (Fig. 3); the simulation of the Volume model is presented more concisely, without a figure. The Appendix concludes with a summary of our test for robustness.

\section{Assumptions}

- Cell shape is that of a right circular cylinder with hemispherical polar caps

- Cell interdivision time $\tau$ follows a Pearson type III distribution, $\mathrm{CV}=20 \%$

- Proportion of nonconstricting cells $=90 \%$

- Mean cell diameter $\bar{D}=0.440 \mu \mathrm{m}, \mathrm{CV}=5 \%$

For the Length model

- Cell length determines the timing of cell-cycle events

- Mean cell length at age $a, \bar{L}(a)$, increases exponentially with cell age; $L(a)$ is normally distributed about $\vec{L}(a)$, CV at controlled cell-cycle event $=7 \cdot 5 \%$, $\bar{L}=1.32 \mu \mathrm{m}$

- Mean cell volume at age $a, \bar{V}(a)$, is determined by cell geometry; $V(a)$ is normally distributed about $\bar{V}(a), \mathrm{CV}$ due to experimental error $=10 \%$

- Mean cell diameter decreases linearly with $L$; diameter-length correlation $\rho=-0 \cdot 12, D$ is normally distributed about $\bar{D}$

For the Volume model

- Cell volume determines the timing of cell-cycle events

- Mean cell volume at age $a, \bar{V}(a)$, increases exponentially with cell age; $V(a)$ is normally distributed about $\bar{V}(a), \mathrm{CV}$ at controlled cell-cycle event $=10 \%$, $\bar{V}=0 \cdot 181 \mu \mathrm{m}^{3}$

- Mean cell length at age $a, \bar{L}(a)$, is determined by cell geometry; $L(a)$ is normally distributed about $\bar{L}(a)$, CV due to experimental error $=7.5 \%$

- Mean cell diameter is independent of $V ; D$ is normally distributed about $\bar{D}$

\section{Implementation}

The simulations were carried out on a population of $10^{6}$ cells. 


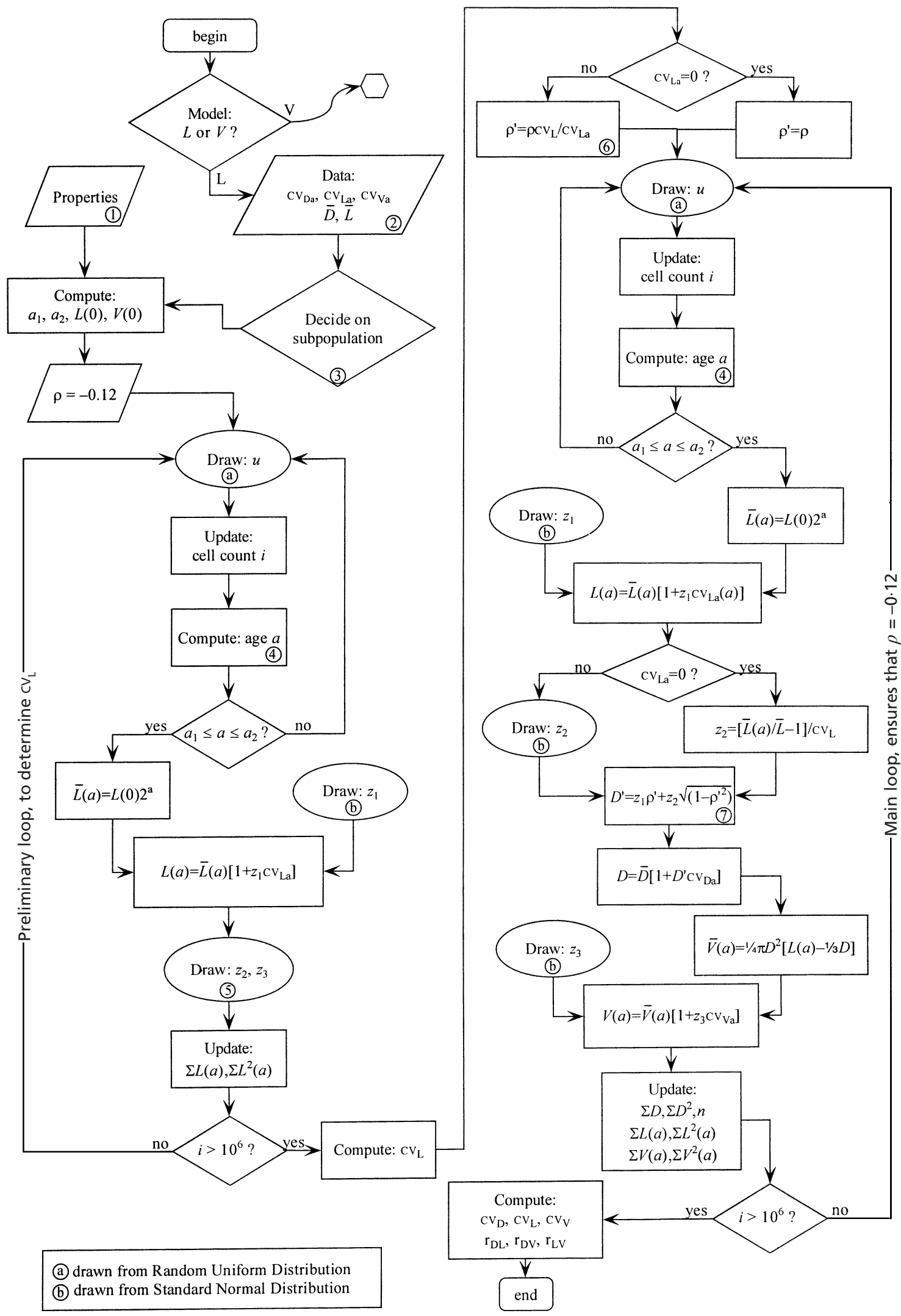

Fig. 3. Flow chart of the simulation process for the Length model. 
The Length model. To be able to set the diameter-length correlation in advance of the simulation it is necessary to determine the value of the coefficient of variation of cell length $\mathrm{CV}_{\mathrm{L}}$ : once this is known, one can ensure that $\rho$ will indeed be as required (see below). The value of $\mathrm{Cv}_{\mathrm{L}}$ is computed in a preliminary simulation loop, as illustrated on the left-hand side of Fig. 3, which we now describe.

(1) The simulation begins by a decision as to the model. The properties of the Length model can be quantified as follows.

- Cell length $L$ determines the timing of all cell-cycle events and its growth is exponential: $\bar{L}(a)=L(0) 2^{a}$, with $a$ in units of the mean interdivision time $\bar{\tau}$

- Cell diameter $D$ decreases with $L(\rho<0$, here we use the value $-0 \cdot 12$ given by Vardi \& Grover, 1992) up to constriction. The model does not specify the behaviour of constricting cells nor is there much experimental data in that range, and we make no attempt to simulate cell growth beyond constriction.

- Cell volume $V$ is determined by cell geometry (right circular cylinder with hemispherical polar caps) $: V=$ $\frac{1}{4} \pi D^{2}\left(L-\frac{1}{3} D\right)$

The distribution of interdivision times $f(\tau)$, and so also the steady-state age distribution $v(a)$, are Pearson type III :

$f(\tau)=\frac{\tau^{g-1} \mathrm{e}^{-\tau / s}}{s^{g} \Gamma(g)}$ and $v(a)=\frac{2 \mu \mathrm{e}^{-\mu a}}{s^{g} \Gamma(g)} \int_{a}^{\infty} \tau^{g-1} \mathrm{e}^{-\tau / s} \mathrm{~d} \tau$,

where $g=\frac{1}{\mathrm{cv}_{\tau}^{2}}, \quad s=\frac{\bar{\tau}}{g}, \quad \mu=\frac{2^{1 / g}-1}{s}$

(2) The simulations were carried out with parameter values based on actual data wherever possible: $\mathrm{CV}_{\mathrm{Da}}=0.05, \quad \mathrm{CV}_{\mathrm{La}}=0.075, \quad \mathrm{CV}_{\mathrm{Va}}=0 \cdot 10, \quad \mathrm{CV}_{\tau}=0 \cdot 20$, $\bar{D}=0.440 \mu \mathrm{m}, \bar{L}=1.32 \mu \mathrm{m}$, where $\mathrm{CV}_{\mathrm{Xa}}$ denotes the coefficient of variation of dimension $X$ in cells of age $a$, and $\bar{L}$ refers to the nonconstricting subpopulation only.

(3) At this point, a decision is taken as to the subpopulation to simulate. The various subpopulations were defined to correspond to those observed empirically (Vardi \& Grover, 1992), as follows.

- Dividing cells: experimentally, obtained from the deeply constricted cells, chosen so that the width of the constricted region $\xi$ did not exceed 0.50 of the mean width of the prospective daughter cells, sample size 637 out of a total of 55269 ; in simulation, oldest $1 \cdot 15 \%$ of the population

- Newborn cells: experimentally, the prospective daughters of the dividing cells, sample size 1274; in simulation, youngest $2.31 \%$ of the population

- Nonconstricting cells: experimentally, relative $\xi>0 \cdot 78$, sample size 50796 ; in simulation, youngest $91.9 \%$ of the population

- Onset of constriction: experimentally, relative $\xi$ in the inclusive range $0 \cdot 65-0 \cdot 78$, sample size 688 ; in simulation, youngest $1 \cdot 24 \%$ of the constricting cell population

The age of the youngest cell within a particular subpopulation is labelled $a_{1}$ and the oldest $a_{2}$, and these were determined from $\int_{0}^{a_{1}} v(a) \mathrm{d} a=$ proportion of cells younger than those of the subpopulation, and $\int_{a_{1}}^{a_{2}} v(a) \mathrm{d} a$ $=$ proportion of cells within the subpopulation. $L(0)$ and $V(0)$ are given by

$L(0)=\bar{L} \frac{\int_{0}^{a_{\mathrm{c}}} v(a) \mathrm{d} a}{\int_{0}^{a_{\mathrm{c}}} 2^{a} v(a) \mathrm{d} a}$ and $V(0)=\frac{1}{4} \pi \bar{D}^{2}\left(L(0)-\frac{1}{3} \bar{D}\right)$,

where $a_{\mathrm{c}}$ is the value of $a_{2}$ in the subpopulation of nonconstricting cells (in which, of course, $a_{1}=0$ ).

(4) A random number $u$ is drawn from a standard uniform distribution. Cell age $a$ is computed from $\int_{0}^{a} v(a) \mathrm{d} a=u$. If it turns out that the cell is not a member of the subpopulation being simulated (that is, if $a<a_{1}$ or $a>a_{2}$ ), we reject it and repeat the draw. If it does belong to the appropriate subpopulation, we proceed by computing the mean cell length at that age $L(a)$ from $L(a)=$ $L(0) 2^{a}$ and then drawing a second random number $z_{1}$, this time from a standard normal distribution. We have assumed that the length of a particular cell at any age $L(a)$ is normally distributed about its mean at that age $\bar{L}(a)$, and so $L(a)=\bar{L}(a)\left[1+z_{1} \mathrm{CV}_{\mathrm{La}}\right]$, where $\mathrm{CV}_{\mathrm{La}}$ is the coefficient of variation of cell length at age $a$, a fixed value, part of the input data, and set at 0.075.

(5) Two more random numbers $z_{2}$ and $z_{3}$ are drawn from the standard normal distribution and discarded; this ensures that the main loop (right-hand side of Fig. 3) produces the same cell sample as the preliminary one (left-hand side). Various counters are updated, such as $\Sigma L(a)$ and $\Sigma L^{2}(a)$, that will be used to compute $\mathrm{CV}_{\mathrm{L}}$. When the total number of cells examined, including those not part of the subpopulation being studied, reaches one million, we exit the preliminary loop and compute $\mathrm{CV}_{\mathrm{L}}$, the coefficient of variation of length in the entire sample. This is to be distinguished from $\mathrm{CV}_{\mathrm{La}}$ which, of necessity, is always smaller than $\mathrm{Cv}_{\mathrm{L}}$. We are now ready for the main simulation process.

(6) After resetting the various counters, we compute an interim variable $\rho^{\prime}=\rho \mathrm{CV}_{\mathrm{L}} / \mathrm{CV}_{\mathrm{La}}$. This is done to ensure that the overall correlation between $D$ and $L$ is $\rho$ (see below). As stated previously, the parameter values used are based on actual data wherever possible. Nonetheless, in order to test the robustness of our conclusions, we repeated the simulations for a large range of parameter values, as listed under the subheading Robustness towards the end of this Appendix. In particular, we also tested $\mathrm{CV}_{\mathrm{La}}=0$, and the extra decision boxes in the main loop were inserted in order to cover that condition. 
Apart from this, the main loop is very similar to the preliminary loop down to the calculation of $L(a)$. At that stage we draw a third random number $z_{2}$ (or compute it, in the case $\left.\mathrm{CV}_{\mathrm{La}}=0\right)$ and define $D^{\prime}$ as $D^{\prime}=z_{1} \rho^{\prime}+$ $z_{2} \sqrt{1-\rho^{\prime 2}}$.

(7) Because $z_{1}$ and $z_{2}$ have mean 0 and variance 1 and are mutually independent, $D^{\prime}$ also has mean 0 and variance 1. Furthermore, the correlation between $D^{\prime}$ and $z_{1}$, and hence between $D^{\prime}$ and $L(a)$, is $\rho^{\prime}$. Since $\rho^{\prime}=\rho \mathrm{CV}_{\mathrm{L}} / \mathrm{CV}_{\mathrm{La}}$, the correlation between $D^{\prime}$ and the overall $L$ is simply $\rho$, as required. Because $D$ is assumed to be normally distributed about $\bar{D}$ with a coefficient of variation $\mathrm{CV}_{\mathrm{Da}}$ independent of cell age, $D$ is given by $D=$ $\bar{D}\left[1+D^{\prime} \mathrm{CV}_{\mathrm{Da}}\right]$; this assures that $D$ has a mean of $\bar{D}$, a coefficient of variation of $\mathrm{CV}_{\mathrm{Da}}$ and a correlation with $L$ of $\rho$ via $D^{\prime}$. The mean volume at that age $\bar{V}(a)$ is computed from the assumed cell shape of a right circular cylinder with hemispherical polar caps: $\bar{V}(a)=$ $\frac{1}{4} \pi D^{2}\left[L(a)-\frac{1}{3} D\right]$. Since $V(a)$ is taken to be normally distributed about $\bar{V}(a)$ with a fixed coefficient of variation of $\mathrm{CV}_{\text {Va }}$, a fourth random number $z_{3}$ is used to compute it: $V(a)=\bar{V}(a)\left[1+z_{3} \mathrm{CV}_{\mathrm{Va}}\right]$.

At this stage we update the various counters of the main loop, $\Sigma D, \Sigma D^{2}$, sample size $n, \Sigma L(a), \Sigma L^{2}(a), \Sigma V(a)$ and $\Sigma V^{2}(a)$, and check to see whether the total number of cells examined in this loop, including those not part of the subpopulation being studied, has reached one million. When it has, we exit the main loop and compute the final output data: the coefficients of variation $\mathrm{CV}_{\mathrm{D}}$, $\mathrm{CV}_{\mathrm{L}}$ and $\mathrm{CV}_{\mathrm{V}}$, and the correlation coefficients $r_{\mathrm{DL}}, r_{\mathrm{DV}}$ and $r_{\mathrm{LV}}$.

The Volume model. The Volume model, as mentioned above, is much simpler. In particular, there is no preliminary loop and no need for $\rho^{\prime}$. A random number $u$ is drawn from a standard uniform distribution and cell age $a$ determined as in the Length model. For cells belonging to the subpopulation being studied, $\bar{V}(a)$ is computed from $\bar{V}(a)=V(0) 2^{a}$. A second random number $z_{1}$, this time drawn from a standard normal distribution, is used to compute $V(a): V(a)=$ $\bar{V}(a)\left[1+z_{1} \mathrm{CV}_{\mathrm{Va}}\right]$. A third random number $z_{2}$, again from a standard normal distribution, is drawn and $D$ computed from $D=\bar{D}\left[1+z_{2} \mathrm{Cv}_{\mathrm{Da}}\right] . \bar{L}(a)$ is then obtained from the assumed cell geometry: $\bar{L}(a)=$ $4 \mathrm{~V}(a) / \pi D^{2}+\frac{1}{3} D$. Finally, a fourth random number $z_{3}$, once again from a standard normal distribution, is used to get $L(a): L(a)=\bar{L}(a)\left[1+z_{3} \mathrm{CV}_{\mathrm{La}}\right]$. The various counters are updated, as in the case of the Length model, and when the total number of cells drawn has reached one million, we exit the loop and compute the three coefficients of variation and the three correlation coefficients.

\section{Robustness}

The results reported here were obtained with the parameters and assumptions listed above, but many other combinations were tested. In no case were the implications or inferences affected, although the actual numerical output of the simulations did differ somewhat with each combination, usually by less than $1 \%$.

- Proportion of constricting cells : 80-95\%

- $\mathrm{CV}$ of interdivision time: $0-25 \%$

- $\mathrm{CV}$ of cell diameter : $2 \cdot 5-10 \%$

- $\mathrm{CV}$ at controlled cell-cycle event ( $V$ for the Volume model, $L$ for the Length model) : 0-20\%

- CV due to experimental error ( $L$ in the Volume model, $V$ in the Length model) : 0-20\%

- Cell shape: right circular cylinder without polar caps

- Cell growth $[\bar{V}(a)$ in the Volume model, $\bar{L}(a)$ in the Length model]: increases linearly with cell age

- Cell interdivision time: follows a normal distribution

- Sample size: $10^{5}-10^{7}$ cells

- Random number generation: three different seeds

\section{ACKNOWLEDGEMENTS}

The authors wish to express their thanks to Charles E. Helmstetter for providing us with membrane-eluted baby cells, to Norbert Vischer for developing some of the digitalacquiring software, and to Arieh Zaritsky for interesting discussions during the early phase of this project.

\section{REFERENCES}

Boye, E., Stokke, T., Kleckner, N. \& Skarstad, K. (1996). Coordinating DNA replication initiation with cell growth: differential roles for DnaA and SeqA proteins. Proc Natl Acad Sci U S A 93, 12206-12211.

Cooper, S. (1989). The constrained hoop: an explanation of the overshoot in cell length during a shift-up of Escherichia coli. J Bacteriol 171, 5239-5243.

Cooper, S. (1991). Bacterial Growth and Division: Biochemistry and Regulation of Prokaryotic and Eukaryotic Division Cycles, p. 222. San Diego: Academic Press.

Donachie, W. D. \& Begg, K. J. (1970). Growth of the bacterial cell. Nature 227, 1220-1224.

Donachie, W. D., Begg, K. J. \& Vicente, M. (1976). Cell length, cell growth, and cell division. Nature 264, 328-333.

Hansen, F. G., Christensen, B. B. \& Atlung, T. (1991). The initiator titration model: computer simulation of chromosome and minichromosome control. Res Microbiol 142, 161-167.

Harvey, R. J. \& Marr, A. G. (1966). Measurement of size distributions of bacterial cells. J Bacteriol 92, 805-811.

Helmstetter, C. E. \& Cooper, S. (1968). DNA synthesis during the division cycle of rapidly growing Escherichia coli B/r. J Mol Biol 31, 507-518.

Koch, A. L. \& Schaechter, M. (1962). A model for statistics of the cell division process. J Gen Microbiol 29, 435-454.

Pritchard, R. H., Barth, P. T. \& Collins, J. (1969). Control of DNA synthesis in bacteria. In Microbial Growth (Society for General Microbiology Symposium 19), pp. 263-297. Edited by P. Meadow \& S. J. Pirt. Cambridge: Cambridge University Press.

Rosenberger, R. F., Grover, N. B., Zaritsky, A. \& Woldringh, C. L. (1978). Surface growth in rod-shaped bacteria. J Theor Biol 73, $711-721$. 
Schaechter, M., Maaløe, O. \& Kjeldgaard, N. O. (1958). Dependency on medium and temperature of cell size and chemical composition during balanced growth of Salmonella typhimurium. J Gen Microbiol 19, 592-606.

Schaechter, M., Williamson, J. P., Hood, J. R., Jr \& Koch, A. L. (1962). Growth, cell and nuclear divisions in some bacteria. J Gen Microbiol 29, 421-434.

Trueba, F. J. \& Woldringh, C. L. (1980). Changes in cell diameter during the division cycle of Escherichia coli. J Bacteriol 142, 869-878.

Van Helvoort, J. M. L. M., Huls, P. G., Vischer, N. O. E. \& Woldringh, C. L. (1998). Fused nucleoids resegregate faster than cell elongation in Escherichia coli $p b p B$ (Ts) filaments after release from chloramphenicol inhibition. Microbiology 144, 1309-1317.

Vardi, E. (1990). Examination of Models Describing the Kinetics of Cell Growth in Escherichia coli. PhD thesis, Hebrew University, Jerusalem (in Hebrew).

Vardi, E. \& Grover, N. B. (1992). Aggregation of Escherichia coli $\mathrm{B} / \mathrm{r}$ A during agar filtration: effect on morphometric measurements. Cytometry 13, 540-544.

Vardi, E. \& Grover, N. B. (1993). Shape changes in Escherichia coli $\mathrm{B} / \mathrm{r}$ A during agar filtration. Cytometry 14, 173-178.

Woldringh, C. L., de Jong, M. A., van den Berg, W. \& Koppes, L. J. H. (1977). Morphological analysis of the division cycle of two
Escherichia coli substrains during slow growth. J Bacteriol 131, 270-279.

Woldringh, C. L., Grover, N. B., Rosenberger, R. F. \& Zaritsky, A. (1980). Dimensional rearrangement of rod-shaped bacteria following nutritional shift-up. II. Experiments with Escherichia coli B/r. J Theor Biol 86, 441-454.

Woldringh, C. L., Mulder, E., Valkenburg, J. A. C., Wientjes, F. B., Zaritsky, A. \& Nanninga, N. (1990). Role of the nucleoid in the toporegulation of division. Res Microbiol 141, 39-49.

Woldringh, C. L., Zaritsky, A. \& Grover, N. B. (1994). Nucleoid partitioning and the division plane in Escherichia coli. J Bacteriol 176, 6030-6038.

Zar, J. H. (1999). Biostatistical Analysis, 4th edn, p. 141. Upper Saddle River, NJ: Prentice Hall.

Zaritsky, A., Woldringh, C. L., Grover, N. B., Naaman, J. \& Rosenberger, R. F. (1982). Growth and form in bacteria. Comments Mol Cell Biophys 1, 237-260.

Zaritsky, A., Woldringh, C. L., Helmstetter, C. E. \& Grover, N. B. (1993). Dimensional rearrangement of Escherichia coli $\mathrm{B} / \mathrm{r}$ cells during a nutritional shift-down. J Gen Microbiol 139, 2711-2714.

Received 5 July 2000; revised 29 August 2000; accepted 8 September 2000. 